\title{
Efectos de una suplementación energética en base a maíz roleado sobre las características de calidad de la canal y la incidencia de corte oscuro en novillos
}

\author{
Effects of a high energy supplementation based on flaked corn on carcass quality traits and the \\ incidence of dark cutting meat in steers.
}

\author{
C Gallo ${ }^{a}$, A Apaoblaza ${ }^{\mathrm{b}}$, RG Pulido ${ }^{\mathrm{a}}$, N Jerez-Timaure ${ }^{\mathrm{c}}$ \\ ${ }^{a}$ Instituto de Ciencia Animal, Facultad de Ciencias Veterinarias, Universidad Austral de Chile, Valdivia, Chile. \\ bPrograma Magíster en Ciencias, mención Salud Animal, Escuela de Graduados, Facultad de Ciencias Veterinarias, \\ Universidad Austral de Chile, Valdivia, Chile.
}

'Departamento de Zootecnia, Facultad de Agronomía, Universidad del Zulia Núcleo Agropecuario, Maracaibo, Venezuela.

\begin{abstract}
SUMMARY
The aim of this study was to determine the effects of a high energy supplementation based on flaked corn and of the duration of this supplementation, on the concentrations of muscle and liver glycogen and on carcass meat quality characteristics related to dark cutting in steers grazing pasture during the fattening stage. Seventy two steers of dairy origin (Red and Black Friesians), with an initial live weight between 460 and $520 \mathrm{~kg}$ were used. Within each treatment (36 steers with and 36 without high energy supplementation), the steers were assigned at random to one of four time lengths of supplementation $(7,14,21$ and 28 días, 9 steers each). The statystical model used was completely at random with a $2 \times 4$ factorial ( 2 treatments and 4 time lengths of treatment); the interaction diet $\mathrm{x}$ duration of the supplementation, the age by dental chronometry and the initial weight of the steers as a covariable were also included. In general, the supplementation resulted in better carcass quality characteristics such as weight, proportional dressing yield, loin eye area and fat cover. Although mean concentrations of muscle glycogen did not differ between treatments, the high energy supplementation reduced the presentation of dark cutters as evaluated through color and $\mathrm{pH}$ in Longissimus thoracis muscle.
\end{abstract}

Key words: steers, energy supplementation, carcasses, $\mathrm{pH}$.

\section{RESUMEN}

El objetivo de esta investigación fue determinar el efecto de una suplementación con concentrado energético, en base a maíz roleado, y de la duración de la suplementación durante la etapa final de engorde a pradera de novillos sobre las concentraciones de glucógeno muscular y hepático, y sobre las características de calidad de la canal y la carne relacionadas con corte oscuro. Se utilizaron 72 novillos de origen lechero (Overo Negro y Colorado), con peso inicial entre 460 y $520 \mathrm{~kg}$. Dentro de cada tratamiento los 36 novillos con y sin suplementación fueron asignados al azar a uno de cuatro tiempos de duración del experimento (7, 14, 21 y 28 días, 9 novillos cada uno). El modelo estadístico usado fue completamente al azar con arreglo factorial 2x4 (2 tratamientos y 4 duraciones), se incluyó además, la interacción dieta x duración de la suplementación, la edad por cronometría dentaria y como covariable el peso inicial de los novillos. La suplementación en base a maíz roleado permitió mejorar características de la canal como peso, rendimiento centesimal, área del ojo del lomo y cobertura grasa. Aunque las concentraciones promedio de glucógeno muscular no difirieron entre tratamientos, la suplementación con maíz roleado ayudó a prevenir la presencia de corte oscuro, evaluada por color y pH del músculo Longissimus thoracis.

Palabras clave: novillos, suplementación energética, canales, pH.

\section{INTRODUCCIÓN}

En bovinos destinados a producir carne, una disminución del contenido de glucógeno muscular antes del beneficio resulta en una baja producción de ácido láctico post mórtem y por ende en carnes con $\mathrm{pH}$ alto $(\mathrm{pH}$ $\geq 5,8$ ), alta capacidad de retención de agua y un color oscuro poco atractivo a la vista (Hood y Tarrant 1980); esta condición es conocida como carnes DFD (dark, firm, and dry, por sus siglas en inglés) o carnes de corte oscu-

Aceptado: 30.05.2013.

* cgallo@uach.cl ro en español. La carne DFD o corte oscuro hoy en día permanece como un importante problema en la industria cárnica de muchos países (Brown y col 1990, Page y col 2001, Gallo 2004, Leyva-García y col 2012).

En Chile, el corte oscuro es un problema serio de calidad, cuya incidencia fluctúa entre $4 \%$ y $10 \%$ generalmente, pero en épocas críticas o en ciertos grupos de animales puede llegar a $25 \%$ y más, especialmente en novillos (Gallo 2004, 2009). El pH elevado limita el uso de la carne para el envasado al vacío, ya que reduce la vida útil de anaquel, y el color oscuro afecta negativamente la aceptabilidad por parte de los consumidores. Por lo anterior, las canales con este problema son devaluadas 
económicamente a nivel de matadero, produciendo pérdidas que han sido estimadas en $15 \%$ en Chile (Vidal y col 2009) y $10 \%$ en México (Leyva-García y col 2012).

La frecuencia de corte oscuro depende de muchos factores tanto propios del animal como del medio ambiente: predisposición genética, sexo, edad, estado nutricional, distancia o tiempo de transporte, época del año, mezcla de ganado de distintas procedencias, tiempo de ayuno y otras en general relacionadas con el estrés (Hood y Tarrant 1980, Tarrant y Sherington 1980, Ferguson y Warner 2008).

El estrés es una inevitable consecuencia del proceso de transferir los bovinos desde el predio al matadero. Los efectos del estrés crónico previo a la faena sobre el contenido de glucógeno muscular y su relación con la condición de carnes de corte oscuro han sido bien documentados en Chile (Hargreaves y col 2003, Gallo 2004, 2009). Sin embargo, las reservas de glucógeno muscular inicial de los novillos también dependen del tipo de alimentación y la cantidad de energía recibida en las etapas finales de la engorda en predio (McVeigh y Tarrant 1982). Immonen y col $\left(2000^{a, b}\right)$ encontraron que el uso de dietas con altas concentraciones de energía en las últimas semanas de engorde podría proteger a los animales de algunos de los muchos factores estresantes que causan una depleción excesiva del glucógeno muscular. A raíz de esta relación con el tipo de alimentación, también se ha encontrado en Chile mayor frecuencia de $\mathrm{pH} \geq 5,8$ en animales que han sido faenados en verano y que consumen sólo pradera que en aquellos faenados en invierno, que consumen avena más ensilaje (Amtmann y col 2006).

El objetivo de este estudio fue determinar el efecto de una suplementación con concentrado energético en base a maíz roleado por cuatro tiempos de suplementación (7, 14, 21 y 28 días) durante la etapa final de engorde a pradera de novillos sobre las concentraciones de glucógeno muscular y hepático, y las características de calidad de la canal y la carne relacionadas con corte oscuro.

\section{MATERIAL Y MÉTODOS}

\section{ANIMALES}

Se utilizaron 72 novillos de origen lechero (Overo Negro y Colorado), de dos, cuatro y seis dientes permanentes, con peso inicial entre 460 y $520 \mathrm{~kg}$. Los 72 novillos fueron identificados con aretes plásticos, se asignaron al azar a dos grupos de 36 novillos cada uno (control y suplementados) y fueron ubicados en un mismo potrero, que fue separado en dos secciones mediante cerco eléctrico. Se utilizó como alimento base una pradera de dos años de ballica anual (Lolium multiflorum), trébol blanco (Trifolium repens) y rosado (Trifolium pratense $L$.). El grupo control sólo tuvo acceso a la pradera y el grupo suplementado tuvo acceso, además de la pradera, a comederos donde recibieron $4 \mathrm{~kg}$ de maíz roleado al vapor ${ }^{1}$ por animal por día, entregado en dos raciones de igual cantidad.

Dentro de cada tratamiento, los 36 novillos fueron a su vez asignados al azar a uno de cuatro tiempos de duración del experimento ( 9 novillos cada uno): 7, 14, 21 y 28 días respectivamente. Al finalizar cada tiempo de duración, se enviaron los 9 novillos suplementados y los 9 novillos controles juntos a la misma planta faenadora para ser sacrificados.

\section{ALIMENTOS}

$\mathrm{Al}$ inicio de cada una de las cuatro semanas de duración del experimento se tomaron muestras de la pradera, para determinar el contenido de materia seca. Esto se realizó según el Método Indirecto de altura comprimida, mediante un plato medidor de forraje o "Risingplate meter" (Ashgrove Plate Meter, Hamilton, New Zealand).

Las características nutricionales de la pradera durante las cuatro semanas del ensayo fueron iguales para los novillos control y suplementados, fluctuando su contenido de energía entre 2,47 y $1,95 \mathrm{Mcal} / \mathrm{kg}$, siendo más alto en la primera y más bajo en la última semana del experimento. El maíz roleado a vapor resulta de someter el grano a una cocción a $100^{\circ} \mathrm{C}$ y 1 atmósfera de presión, por un tiempo determinado (30-40 minutos) hasta alcanzar un contenido de humedad entre 18 y $20 \%$; posteriormente el grano es laminado mediante dos rodillos, con una separación de $0,005 \mathrm{~mm}$, lográndose un importante grado de gelatinización del almidón, para finalmente ser desecado y enfriado ( $c$. $14 \%$ de humedad). Fue adquirido en la agroindustria local en envases de $40 \mathrm{~kg}$ en una sola partida y presentó las siguientes características: proteína total $6,02 \%$, energía metabolizable $3,01 \mathrm{Mcal} / \mathrm{kg}$, fibra cruda $5,5 \%$, extracto etéreo $1,55 \%$, cenizas $1,12 \%$, humedad $14,0 \%$.

\section{PROCEDIMIENTOS REALIZADOS EN LOS ANIMALES}

Se registraron individualmente los pesos de los novillos al inicio del ensayo, al final de cada duración del tratamiento y al arribo a la planta faenadora. Los 18 novillos enviados a faena cada semana ( 9 suplementados y 9 controles) fueron manejados de la misma forma durante la carga, transporte y descarga. Los animales fueron transportados juntos en el mismo camión, que fue conducido por la misma persona cada vez. El tiempo de transporte entre el predio y la planta faenadora fluctuó entre 3 y 5 horas, según las condiciones ambientales; excepto en el grupo en tratamiento por 28 días, en que por problemas técnicos del camión el viaje demoró 5 horas adicionales. Los animales de ambos tratamientos se mantuvieron en un solo grupo en el mismo corral y por similar tiempo (12-16 h), en reposo en la planta faenadora, previo al faenamiento. Los novillos fueron sacrificados según los

\footnotetext{
${ }^{1}$ Copeval S.A.
} 
estándares comerciales (MINAGRI 2009) de una planta faenadora de exportación; inmediatamente después de faenados se registraron los pesos de las canales calientes; éstos fueron usados para el cálculo del rendimiento centesimal tomando como base el peso vivo final en predio.

\section{DETERMINACIÓN DE GLUCÓGENO MUSCULAR Y HEPÁTICO}

Inmediatamente después del sacrificio (entre 15 y 30 min después del desangrado) se tomó una muestra del músculo Semimembranosus (aproximadamente $10 \mathrm{~g}$ ) de cada canal para la determinación del contenido de glucógeno muscular y similar cantidad de tejido hepático para la determinación de glucógeno hepático. Las muestras, previamente identificadas, se congelaron inmediatamente en nitrógeno líquido y se enviaron al Laboratorio de la Facultad de Ciencias Veterinarias de la Universidad de Chile, Santiago, para su análisis. El análisis de glucógeno consistió en extraer aproximadamente $1 \mathrm{~g}$ de tejido por muestra, que fue homogenizado con $10 \mathrm{ml}$ de HCL al $1 \mathrm{~N}$ y posteriormente hidrolizado durante 2 horas a $100^{\circ} \mathrm{C}$. Paralelamente una muestra de aproximadamente $1 \mathrm{~g}$ de tejido se homogenizó en amortiguador Tris $20 \mathrm{mM}, \mathrm{pH} 7,5$. Alícuotas apropiadas de cada muestra (por ej. 5, 10, $25 \mathrm{~mL}$ ) se usaron para determinar la glucosa oxidasa. En cada oportunidad se preparó una curva de calibración usando un estándar de glucosa $1 \mathrm{mg} / \mathrm{mL}$ con al menos 5 puntos. La concentración de glucógeno de cada muestra se obtuvo sacando la diferencia entre los valores del glucógeno hidrolizado (glucógeno intracelular más el extracelular) y el glucógeno control (glucógeno extracelular). Los valores se expresaron como mmol de glucosa por gramo de tejido (peso húmedo) usando el promedio de al menos dos determinaciones.

\section{EVALUACIÓN DE LA CANAL}

Las canales fueron refrigeradas entre $0^{\circ}$ y $4^{\circ} \mathrm{C}$ por 24 h. Por razones comerciales de la planta las mediciones de pH sólo pudieron ser realizadas en 33 canales de novillos control y 31 de los suplementados. El pH del músculo se determinó por medio de un peachímetro con electrodo de pincho (Hanna Instruments) introducido al centro del músculo Longissimus thoracis, seccionado transversalmente a la altura de la $9^{a}$ costilla. Sobre la superficie de corte del L. thoracis se midió: color del músculo (espectrocolorímetro HUNTERLAB); área del ojo del lomo (AOL), dibujando el contorno del músculo sobre papel milimetrado transparente $\left(\mathrm{cm}^{2)}\right.$; grado de marmoreo y color por apreciación visual, utilizando la pauta de clasificación de canales bovinas de AUS-MEAT (1999). El espesor de grasa dorsal (EGD) se determinó a nivel de la $9^{\mathrm{a}}$ costilla midiendo con un piedemetro $(\mathrm{mm})$ el espesor de la grasa subcutánea en un punto ubicado en la línea media sobre la parte central del músculo L. thoracis. La cobertura de grasa se evaluó por apreciación visual de la canal completa, según la Norma Chilena de Tipificación de Canales de Bovino (INN 2002).

\section{ANÁLISIS ESTADÍSTICO}

Los datos fueron analizados con el paquete estadístico SAS (SAS 2005). Se utilizó el PROC GLM de SAS para el análisis de varianza-covarianza con un modelo completamente al azar con arreglo factorial $2 \times 4$ : tratamiento de suplementación (suplementado vs. control) y duración del tratamiento (7, 14, 21 y 28 días). En el modelo estadístico se incluyó además, la interacción suplementación x duración del tratamiento y como covariable se consideró el peso al inicio del ensayo con un total de nueve repeticiones por tratamiento.

Debido a la variación encontrada en la cronometría dentaria (2, 4 y 6 dientes permanentes) de los novillos al momento de su observación en matadero, se realizó un análisis preliminar que incluyó esta variable, además de las ya mencionadas. El análisis de varianza-covarianza mostró efectos significativos de la edad por cronometría dentaria sólo sobre las variables respuestas $\mathrm{L}^{*}, \mathrm{a}^{*}, \mathrm{~b}^{*}$, $\mathrm{pH}$ y color muscular. Para el resto de las variables, por no haber un efecto significativo $(\mathrm{P}>0,05)$, se omitió la inclusión del efecto de la edad por cronometría dentaria. Cuando los efectos resultaron significativos $(\mathrm{P}<0,05)$ se obtuvieron las medias mínima cuadráticas usando la opción LSMEANS y PDIFF del SAS.

\section{RESULTADOS}

\section{VARIABLES RELACIONADAS CON EL DESEMPEÑO PRODUCTIVO DURANTE LA ENGORDA}

El análisis de varianza-covarianza reveló que el tratamiento de suplementación energética no afectó $(\mathrm{P}>0,05)$ las variables peso vivo final, ganancia diaria de peso, ganancia total, ni pérdida de peso durante el transporte. La duración de la suplementación (cuadro 1) afectó el peso vivo final $(\mathrm{P}<0,0001)$, la ganancia total de peso $(\mathrm{P}<0,0001)$, y la pérdida de peso durante el transporte $(\mathrm{P}<0,0001)$; sin embargo, la ganancia diaria de peso no resultó afectada $(\mathrm{P}>0,05)$ por la duración de la suplementación. El efecto de la interacción suplementación energética $\mathrm{x}$ tiempo de suplementación resultó no significativo $(\mathrm{P}>0,05)$ para todas las variables estudiadas, por lo que los valores promedios se presentan sólo para los efectos simples (cuadro 1).

El peso vivo final y la ganancia total durante el engorde resultaron mayores en los novillos con 28 días de suplementación, con $25,57 \mathrm{~kg}$ de peso vivo final más que los suplementados durante 21 días $(\mathrm{P}<0,05) ; 30,07 \mathrm{~kg}$ más que los suplementados durante 14 días y $35,47 \mathrm{~kg}$ más que los suplementados durante 7 días (cuadro 1). Sin embargo, el grupo de novillos suplementado por 28 
días también resultó con las mayores pérdidas de peso durante el transporte. El efecto de la duración de la suplementación sólo resultó significativo $(\mathrm{P}=0,01)$ para el rendimiento de canal, encontrando que los novillos con mayor tiempo de suplementación (28 días) resultaron con el promedio más bajo $(46,86 \pm 1,70)$ (cuadro 1$)$.

\section{VARIABLES DE LA CANAL}

El análisis de varianza-covarianza reveló que el tratamiento de suplementación energética (cuadro 2) afectó significativamente el peso de canal $(\mathrm{P}=0,01)$, el rendimiento centesimal de canal $(\mathrm{P}=0,02)$, el AOL $(\mathrm{P}=0,02)$, la cobertura de grasa $(\mathrm{P}=0,0003)$ y el EGD $(\mathrm{P}=0,05)$. Los novillos suplementados tuvieron $8,82 \mathrm{~kg}$ más de canal que los controles, reflejándose en un 1,32 \% más de rendimiento de canal a favor de éstos. De igual modo la suplementación aumentó el AOL en 4,12 cm², el EGD en $0,79 \mathrm{~mm}$ y también se observó una cobertura de grasa significativamente mayor en los novillos con suplementación energética (cuadro 2). Sin embargo, el marmoreo resultó similar para ambos tratamientos $(\mathrm{P}>0,05)$, ubicándose en la escala cercana a 1 , que correspondería a un contenido de trazas a prácticamente desprovisto de grasa intramuscular.

\section{CONTENIDO DE GLUCÓGENO, pH Y COLOR MUSCULAR}

El análisis de varianza-covarianza mostró efecto significativo del tratamiento de suplementación (cuadro 3) sobre el $\mathrm{pH}$ medido a las $24 \mathrm{~h}$ postmórtem $(\mathrm{P}=0,0007)$; así mismo sobre el promedio de luminosidad $\mathrm{L}^{*}(\mathrm{P}=$ $0,001)$; intensidad de rojo $\mathrm{a}^{*}(\mathrm{P}=0,0005)$, intensidad de amarillo $b^{*}(\mathrm{P}=0,007)$ y el color muscular medido con la pauta de AUS-MEAT $(P=0,009)$. Las carnes provenientes de animales suplementados presentaron un promedio de $\mathrm{pH}$ más bajo y promedios de $\mathrm{L}^{*}, \mathrm{a}^{*}$ y $\mathrm{b}^{*}$ más altos

Cuadro 1. Efecto de la duración de la suplementación energética sobre las variables peso vivo y ganancia de peso en novillos engordados a pradera.

Effect of duration of energy supplementation on live weight and live weight gain in steers fattened on pasture.

\begin{tabular}{|c|c|c|c|c|c|}
\hline \multirow[t]{2}{*}{ Variables } & \multicolumn{4}{|c|}{ Duración de la suplementación } & \multirow[b]{2}{*}{ Valor $\mathrm{P}$} \\
\hline & 7 días $(\mathrm{n}=18)$ & 14 días $(\mathrm{n}=17)$ & 21 días $(\mathrm{n}=18)$ & 28 días $(\mathrm{n}=18)$ & \\
\hline Peso final vivo $^{1}$ & $546,25 \pm 6,04^{a}$ & $551,65 \pm 5,06^{\mathrm{a}}$ & $556,15 \pm 5,13^{\mathrm{a}}$ & $581,72 \pm 4,82^{\mathrm{b}}$ & $<0,0001$ \\
\hline Ganancia durante el engorde ${ }^{2}$ & $25,01 \pm 6,04^{\mathrm{a}}$ & $30,40 \pm 5,06^{\mathrm{a}}$ & $34,89 \pm 5,13^{a}$ & $60,47 \pm 4,82^{\mathrm{b}}$ & $<0,0001$ \\
\hline Pérdida peso durante el transporte ${ }^{3}$ & $41,28 \pm 3,72^{\mathrm{c}}$ & $31,20 \pm 3,12^{\mathrm{a}}$ & $37,62 \pm 3,16^{\mathrm{ac}}$ & $49,75 \pm 2,97^{b}$ & 0,0001 \\
\hline Ganancia diaria de peso & $1,44 \pm 0,25$ & $1,45 \pm 0,20$ & $1,24 \pm 0,21$ & $1,71 \pm 0,19$ & ns \\
\hline Peso canal caliente, $\mathrm{kg}$ & $262,34 \pm 4,28$ & $267,09 \pm 3,59$ & $270,6 \pm 3,64$ & $268,59 \pm 3,42$ & $\mathrm{~ns}$ \\
\hline Rendimiento canal $\%$ & $48,02 \pm 0,78^{c}$ & $48,40 \pm 0,65^{\mathrm{a}}$ & $48,57 \pm 0,66^{\mathrm{ac}}$ & $46,14 \pm 0,62^{b}$ & 0,01 \\
\hline
\end{tabular}

${ }^{1}$ : Peso tomado en el predio antes del envío a planta faenadora.

2: Obtenido por diferencia entre el peso final y el peso al inicio del experimento.

3: Diferencia de peso entre el peso vivo al llegar a planta faenadora y el peso final al salir del predio.

4: Porcentaje de peso.

Cuadro 2. Efecto de la suplementación energética sobre las características de la canal de novillos engordados a pradera. Effect of a high energy supplementation on carcass traits of steers fattened on pasture.

\begin{tabular}{lrcc}
\hline Variables & \multicolumn{2}{c}{ Tratamiento } & \multirow{2}{*}{ Valor P } \\
\cline { 2 - 3 } & Control $(\mathrm{n}=36)$ & Suplementación $(\mathrm{n}=36)$ & 0,01 \\
Peso canal caliente, $\mathrm{kg}$ & $262,96 \pm 2,95$ & $271,78 \pm 3,03$ & 0,02 \\
Rendimiento canal, \% & $47,12 \pm 0,52$ & $48,44 \pm 0,57$ & 0,02 \\
Área del ojo del lomo, cm $^{2}$ & $45,23 \pm 1,44$ & $49,35 \pm 1,64$ & 0,003 \\
Cobertura de grasa $^{1}$ & $0,96 \pm 0,08$ & $1,25 \pm 0,09$ & 0,05 \\
Espesor grasa dorsal, mm $_{\text {Marmoreo }^{2}}$ & $3,06 \pm 0,35$ & $3,85 \pm 0,39$ & $\mathrm{~ns}$ \\
\hline
\end{tabular}

${ }^{1}$ : Evaluación de acuerdo la Norma Chilena de Tipificación de Canales de Bovino (Chile, 2002).

2: Se evaluó usando la pauta de clasificación de canales bovinas de AUS-MEAT (1999).

ns: No significativo $\mathrm{P}>0,05$.

$\mathrm{n}=36$ para Peso Canal Caliente. 
que las carnes provenientes de animales controles. Lo anterior indica que las carnes de novillos suplementados resultaron con una mayor luminosidad (más claras) y una intensidad de rojo y amarillo mayor que las carnes del grupo control. De igual modo, la medición de color según el patrón de AUS-MEAT (1999) confirmó que las carnes provenientes de novillos no suplementados presentaron un color más oscuro a la vista que las de los suplemen- tados. El contenido de glucógeno muscular y hepático no fueron afectados $(\mathrm{P}>0,05)$ por el tratamiento de suplementación, y tampoco el color de la grasa (cuadro 3). En total, de las 33 canales analizadas de novillos controles, 8 presentaron $\mathrm{pH} \geq 5,8$ (24\%): 1, 2, 2 y 3 en los tratamientos por 7, 14, 21 y 28 días respectivamente; en cambio, de las 31 canales examinadas de los novillos suplementados, no hubo ninguna con $\mathrm{pH} \geq 5,8$.

Cuadro 3. Efecto de una suplementación energética sobre el contenido de glucógeno, pH y el color muscular en canales de novillos engordados a pradera.

Effect of a high energy supplementation on glycogen content, $\mathrm{pH}$ and color of muscle in carcasses of steers fattened on pasture.

\begin{tabular}{lrrr}
\hline Variables & \multicolumn{3}{c}{ Tratamiento } \\
\cline { 2 - 3 } & Control $(\mathrm{n}=33)$ & Suplementación $(\mathrm{n}=31)$ & $\mathrm{n}$ \\
\hline Glucógeno muscular $^{1}$ & $32,31 \pm 2,78$ & $33,61 \pm 2,96$ & $\mathrm{~ns}$ \\
Glucógeno hepático $^{1}$ & $84,34 \pm 7,62$ & $92,54 \pm 8,16$ & $\mathrm{~ns}$ \\
pH 24 hr postmortem & $5,69 \pm 0,04$ & $5,50 \pm 0,05$ & 0,0007 \\
L* muscular $^{2}$ & $23,38 \pm 0,43$ & $25,07 \pm 0,49$ & 0,001 \\
a $^{*}$ muscular $^{3}$ & $11,96 \pm 0,33$ & $13,41 \pm 0,37$ & 0,007 \\
b* muscular $^{4}$ & $6,91 \pm 0,23$ & $7,87 \pm 0,26$ & 0,009 \\
Color muscular $^{5}$ & $5,18 \pm 0,11$ & $4,84 \pm 0,12$ & $\mathrm{~ns}$ \\
Color de la grasa $^{6}$ & $5,47 \pm 0,16$ & $5,30 \pm 0,17$ & \\
\hline
\end{tabular}

${ }^{1}: \mathrm{mmol} / \mathrm{gr}$ tejido fresco

2: $\mathrm{L}^{*}=$ intensidad del luz; $100=$ blanco, $0=$ negro.

3: $\mathrm{a}^{*}=$ intensidad de rojo; $-80=$ verde, $100=$ rojo.

4: $\mathrm{b}^{*}=$ intensidad de amarillo; $-50=$ azul, $70=$ amarillo .

5: Se evaluó usando la pauta de clasificación de canales bovinas de AUS-MEAT (1999).

6. Se evaluó usando la pauta de clasificación de canales bovinas de AUS-MEAT (1999).

ns: no significativo $\mathrm{P}>0,05$.

Cuadro 4. Efecto de la duración de una suplementación energética sobre el contenido de glucógeno, el pH y el color muscular en canales de novillos engordados a pradera.

Effect of the duration of a high energy supplementation on glycogen content, $\mathrm{pH}$ and color in the carcasses of steers fattened on pasture.

\begin{tabular}{|c|c|c|c|c|c|}
\hline \multirow[t]{2}{*}{ Variable } & \multicolumn{5}{|c|}{ Duración de la suplementación } \\
\hline & 7 días $(\mathrm{n}=18)$ & 14 días $(\mathrm{n}=17)$ & 21 días $(\mathrm{n}=11)$ & 28 días $(\mathrm{n}=18)$ & Valor P \\
\hline Glucógeno muscular $^{1}$ & $37,03 \pm 4,18^{\mathrm{a}}$ & $35,93 \pm 3,50^{a}$ & $37,10 \pm 3,55^{\mathrm{a}}$ & $21,77 \pm 3,43^{b}$ & 0,0009 \\
\hline Glucógeno hepático ${ }^{1}$ & $78,31 \pm 9,49$ & $89,94 \pm 9,59$ & $105,93 \pm 9,96$ & $79,58 \pm 9,14$ & ns \\
\hline $\mathrm{pH} 24 \mathrm{hr}$ postmortem & $5,59 \pm 0,07$ & $5,52 \pm 0,05$ & $5,61 \pm 0,07$ & $5,66 \pm 0,05$ & ns \\
\hline $\mathrm{L}^{*}$ muscular ${ }^{2}$ & $22,67 \pm 0,65^{\mathrm{a}}$ & $25,31 \pm 0,53^{\mathrm{b}}$ & $23,82 \pm 0,67^{\mathrm{a}}$ & $25,10 \pm 0,51^{\mathrm{b}}$ & 0,002 \\
\hline $\mathrm{a}^{*}$ muscular $^{3}$ & $11,44 \pm 0,50^{\mathrm{a}}$ & $13,31 \pm 0,41^{\mathrm{b}}$ & $14,46 \pm 0,51^{b}$ & $11,52 \pm 0,39^{a}$ & 0,0001 \\
\hline $\mathrm{b}^{*}$ muscular $^{4}$ & $6,61 \pm 0,34^{a}$ & $7,89 \pm 0,8^{\mathrm{b}}$ & $8,34 \pm 0,35^{\mathrm{b}}$ & $6,73 \pm 0,27^{\mathrm{a}}$ & 0,0001 \\
\hline Color muscular ${ }^{5}$ & $5,23 \pm 0,16^{\mathrm{b}}$ & $4,51 \pm 0,13^{\mathrm{a}}$ & $5,03 \pm 0,17^{\mathrm{b}}$ & $5,27 \pm 0,13^{b}$ & 0,0002 \\
\hline Color grasa ${ }^{6}$ & $5,03 \pm 0,23$ & $5,67 \pm 0,19$ & $5,82 \pm 0,24$ & $5,033 \pm 0,18$ & ns \\
\hline
\end{tabular}

${ }^{1}: \mathrm{mmol} / \mathrm{g}$ tejido fresco

2: L*$^{*}=$ intensidad del luz; $100=$ blanco, $0=$ negro.

3: $\mathrm{a}^{*}=$ intensidad de rojo $;-80=$ verde, $100=$ rojo.

4: $\mathrm{b}^{*}=$ intensidad de amarillo; $-50=$ azul, $70=$ amarillo.

5: Se evaluó usando la pauta de clasificación de canales bovinas de AUS-MEAT (1999).

6: Se evaluó usando la pauta de clasificación de canales bovinas de AUS-MEAT (1999).

ns: no significativo $\mathrm{P}>0,05$. 
En el cuadro 4 se muestran las medias cuadráticas según la duración de la suplementación; las concentraciones de glucógeno muscular resultaron similares para los novillos en tratamiento por 7, 14 y 21 días (valores entre 35,93 y $37,10 \mu \mathrm{mol} / \mathrm{g}$ tejido); sin embargo los animales que recibieron 28 días de suplementación mostraron un menor $(21,77 \mu \mathrm{mol} / \mathrm{g})$ contenido de glucógeno muscular postmórtem. Las carnes provenientes de novillos suplementados por 14 y 28 días resultaron con promedios de luminosidad $\left(\mathrm{L}^{*}\right)$ más altos, indicando que son carnes más claras y que tuvieron una mayor intensidad del tono rojo; sin embargo las carnes provenientes de animales suplementados durante 14 y 21 días resultaron con una mayor intensidad en la coloración roja (a) y amarilla (b) que los suplementados durante 7 y 28 días. La apreciación visual de color caracterizó a la carne de novillos suplementados durante 14 días en la escala más baja, que indica un color rojo más claro que el resto. $\mathrm{El} \mathrm{pH}$ a las 24 h postmórtem y el contenido de glucógeno hepático no difirieron $(\mathrm{P}>0,05)$ según la duración de la suplementación. La interacción tratamiento x duración de la suplementación no afectó ninguna de las variables estudiadas.

\section{DISCUSIÓN}

\section{VARIABLES RELACIONADAS CON EL DESEMPEÑO PRODUCTIVO Y LAS CARACTERÍSTICAS DE CANAL}

Con respecto al tiempo de suplementación (cuadro 1), era de esperar que a una mayor exposición al suplemento los novillos aumentaran el peso vivo y la ganancia total, a pesar de no encontrar diferencias en las ganancias diarias de peso. Sin embargo los novillos suplementados con maíz roleado no se diferenciaron de los controles en cuanto al peso vivo final, ganancia diaria de peso, ni pérdida de peso debido a transporte. Estos resultados concuerdan con lo informado por Pulido y col (2009), quienes mostraron que vacas lecheras alimentadas sólo a pradera consumieron 16,1 kg/día en comparación con un promedio de $11,9 \mathrm{~kg} /$ día cuando fueron suplementadas con $6 \mathrm{~kg}$ de concentrado por día $(\mathrm{P}=0,001)$, demostrando una sustitución de pradera por concentrado. Si bien en este estudio no se midió el consumo de pradera, los resultados indicarían que los novillos con suplementación energética sustituyeron pradera por maíz roleado. Por ello, aunque las ganancias de peso fueron similares, los efectos observados en las canales (cuadro 2) se fundamentarían por la mayor cantidad de energía consumida por los novillos suplementados.

La suplementación con maíz roleado mejoró la calidad de la canal de los novillos, los que presentaron un mayor rendimiento centesimal, peso de canal caliente, área del ojo del lomo (AOL), espesor de grasa dorsal (EGD) y cobertura de grasa (cuadro 2) que los novillos controles. Estos resultados concuerdan con el hecho que un nivel de alimentación alto antes del sacrificio tiene un efecto positivo en el engrasamiento del animal y en sus rendimientos (Robelin y Daenicke 1980, Kover y col 1984). Posiblemente, la mayor área del ojo del lomo de los novillos con suplementación energética respecto a los controles en este estudio corresponda al hecho que el origen lechero de los novillos implica una madurez más tardía (Johnson y col 1972), que permite aún un crecimiento muscular en el período de 470 a $550 \mathrm{~kg}$, como el utilizado en el estudio. En base a estos resultados se puede inferir que los animales suplementados utilizaron la energía aportada por el maíz roleado durante la fase de engorda para incrementar la masa muscular y depositar más grasa de cobertura, lo que favoreció un mayor peso en canal y mayor rendimiento en canal.

Aún cuando los novillos con suplementación energética presentaron una mayor cobertura de grasa y consecuentemente un mayor EGD (cuadro 2), esto no se reflejó en una mayor cantidad de grasa intramuscular según la pauta de AUS-MEAT (1999), ya que no se observaron diferencias significativas entre novillos control y con suplementación energética en el grado de marmoreo (cuadro 2 ). Esto se debería a que la grasa intramuscular es depositada de manera más lenta y tardíamente en bovinos de origen lechero (Johnson y col 1972). Por otra parte todos los novillos del presente estudio presentaron cobertura de grasa 1 (escasa), excepto dos del grupo suplementado, que presentaron grado 2; esto significa que con escasa grasa de cobertura no es posible esperar en razas de doble propósito y de origen lechero, como las utilizadas, un mayor grado de marmoreo.

La mayor pérdida de peso durante el transporte encontrada en los novillos en tratamiento durante 28 días (cuadro 1) puede deberse a los problemas ocurridos durante el transporte de estos animales; por una avería mecánica la duración del viaje fue de $5 \mathrm{~h}$ más que en los otros grupos y bajo condiciones de alta temperatura ambiental $\left(38^{\circ} \mathrm{C}\right)$ los animales debieron esperar en el camión. Estas condiciones fundamentan la mayor pérdida de peso por transporte de estos novillos y el menor rendimiento centesimal, coincidentes con mayor deshidratación (Gallo 2009). Tal como se ha demostrado en otros trabajos en Chile, los largos tiempos de transporte y espera en ayuno no sólo producen mayor pérdida de peso vivo por vaciamiento del contenido del digestivo de los bovinos, sino que pérdida de peso de la canal (Gallo y col 2003).

\section{CONTENIDO DE GLUCÓGENO, pH Y COLOR MUSCULAR}

El contenido de glucógeno muscular y hepático no fueron afectados $(\mathrm{P}>0,05)$ por la suplementación (cuadros 3 y 4). Al respecto, Gallo y Lizondo (2000) encontraron en novillos a pradera promedios de $195,9 \mu \mathrm{mol} / \mathrm{g}$ y 92,4 $\mu \mathrm{mol} / \mathrm{g}$ de glucógeno hepático, luego de 3 y 24 h de ayuno posterior a un transporte corto $(3 \mathrm{~h})$; estos valores 
coinciden con los del presente estudio. Las concentraciones de glucógeno hepático fueron 2 a 3 veces superiores a las de glucógeno muscular, cuyos promedios fueron de $32,31 \pm 2,88 \mu \mathrm{mol} / \mathrm{g}$ en los novillos control y de 33,61 \pm $2,96 \mu \mathrm{mol} / \mathrm{g}$ en aquellos con suplementación energética (cuadro 3). Estos valores resultan similares a los de estudios anteriores realizados en Chile (Gallo y Lizondo 2000, Hargreaves y col 2003).

Según Brown y col (1990) concentraciones de glucógeno muscular menores a $27,7 \mu \mathrm{mol} / \mathrm{g}$ ya indican una alta probabilidad de encontrar canales con corte oscuro. Este valor sólo fue obtenido como promedio en los novillos en tratamiento durante 28 días (cuadro 4), que a la vez mostraron el mayor número de canales con $\mathrm{pH}>5,8(\mathrm{n}=3)$; el resto presentó promedios de concentraciones de glucógeno muscular mayores a $35 \mu \mathrm{mol} / \mathrm{g}$. Mencarini (2002) obtuvo 32,9 $\mu \mathrm{mol} / \mathrm{g}$ de glucógeno muscular en novillos de similar genotipo tras $3 \mathrm{~h}$ de transporte, valor similar al del presente estudio. Hay muchos factores estresantes previos a la faena que pueden reducir las reservas de glucógeno muscular (Immonen y col 2000ªb). Como se explicó anteriormente, los novillos control y suplementados por 28 días fueron los que tuvieron mayor tiempo de transporte; tanto este factor como las condiciones de alta temperatura ambiental que sufrieron estos novillos, probablemente produjeron elevados niveles de estrés que comprometieron un gasto de glucógeno muscular mayor que en los otros grupos.

El glucógeno muscular responde en general adecuadamente a una alimentación energética (Gardner y col 2001). Sin embargo, en este caso ni la suplementación ni la mayor duración de ésta, tuvieron un efecto significativo sobre una mayor concentración de glucógeno muscular al momento del sacrificio. Es importante resaltar que el no haber monitoreado las concentraciones de glucógeno muscular in vivo, antes de salir los animales del predio, no permite inferir si realmente el suplemento energético había incrementado las concentraciones de glucógeno muscular durante la engorda. Como sólo se determinó la concentración de glucógeno muscular postmórtem, no se pudo separar el posible efecto positivo de la suplementación energética, de aquellos efectos negativos del manejo antemortem, que tienden a reducir las concentraciones de glucógeno muscular. Considerando los resultados obtenidos en este estudio, parece importante para futuros estudios en esta temática, poder determinar si antes del envío a faena las concentraciones de glucógeno se logran aumentar con una suplementación. Esto es posible de realizar mediante biopsias musculares obtenidas a distintos tiempos en un mismo animal (Immonen y col 2000' ${ }^{\mathrm{b}}$, Gardner y col 2001).

Otro aspecto importante de destacar en el actual ensayo es que por razones comerciales, las mediciones de glucógeno muscular debieron ser realizadas en el músculo Semimembranosus (posta negra) en tanto las mediciones de $\mathrm{pH}$, color objetivo y subjetivo fueron realizadas en el músculo L. thoracis (lomo vetado), el cual es usado como indicador de glucógeno muscular en la mayoría de los estudios. Estos dos músculos presentan distintas sensibilidades en su respuesta al estrés y corte oscuro (Hood y Tarrant 1980) y ello puede haber influido en los resultados. En Chile, Almonacid (2003) encontró que al tomar como indicador del $\mathrm{pH}$ elevado de la canal el lomo vetado a nivel de la $9^{\text {a }}$ costilla (músculo L. thoracis), en la posta negra (Semimembranosus) de los mismos animales, sólo un $50 \%$ tenía $\mathrm{pH}$ elevado.

A pesar de no haber encontrado efecto del tratamiento sobre la concentración de glucógeno muscular, las carnes provenientes de animales suplementados presentaron valores de $\mathrm{pH}$ más bajos y un color muscular más claro (cuadro 3). El efecto observado sobre el pH es similar al registrado por Knee y col (2007) y Del Campo y col (2008). También concuerda con French y col (2001) en el sentido que en general novillos alimentados con pradera alcanzan valores de $\mathrm{pH}$ final más elevados que novillos alimentados con granos. Más aún, todos los valores de $\mathrm{pH}$ de los novillos suplementados fueron normales ( $<$ a 5,8) en tanto, ocho valores de $\mathrm{pH}$ de los novillos controles (alimentados a pradera solamente) no lo fueron. El presente trabajo demuestra que al suplementar novillos a pradera con alimento energético (maíz roleado) se disminuye el riesgo de que sus canales tengan $\mathrm{pH}$ igual o superior a 5,8 .

En el total de 64 canales analizadas en este estudio se encontró un $12,5 \%$ de incidencia de canales con $\mathrm{pH} \geq 5,8$; sin embargo en los novillos control la incidencia de $\mathrm{pH} \geq 5,8$ alcanzó un 24,2 \%. Estas frecuencias de presentación del problema de $\mathrm{pH}$ y corte oscuro son similares a las registradas anteriormente por otros autores en Chile (Hargreaves y col 2003, Gallo 2004). Los mismos autores señalan que el problema aumenta con mayor tiempo de transporte y ayuno previo a la faena.

Los promedios de $\mathrm{L}^{*}, \mathrm{a}^{*} \mathrm{y} \mathrm{b}^{*}$ son significativamente menores en las canales con corte oscuro que en canales con pH normal (Page y col 2001). En el presente estudio la luminosidad del músculo ( $\mathrm{L}^{*}$ ) de los novillos controles fue de 23,38 \pm 0,43 y en los novillos con suplementación energética de 25,07 \pm 0,49 (cuadro 3). Los valores de $\mathrm{L}^{*}$ y a* indican que el músculo era más luminoso en los novillos con suplementación energética y tenía un tenor de rojo más claro que en los novillos controles. Paralelamente se utilizó la pauta de AUS-MEAT (1999) para evaluar el color del músculo y los promedios obtenidos fueron para los controles de 5,18 $\pm 0,11$ y los suplementados 4,84 $\pm 0,12(\mathrm{P}=0,009)$. En conclusión, el color del músculo, tanto objetiva como subjetivamente, resultó más oscuro en los novillos control que en los novillos suplementados con maíz roleado. Los resultados de color son coincidentes con las tendencias encontradas en los estudios realizados por Gallo y Lizondo (2000) y Page y col (2001) respecto a los valores de $\mathrm{L}^{*}, \mathrm{a}^{*} \mathrm{y} \mathrm{b}^{*}$, el $\mathrm{pH}$ y su relación con la presencia con corte oscuro. 
En conclusión, en cuanto a los indicadores de calidad de canal, se puede señalar que la suplementación en base a maíz roleado en la etapa final de la engorda de novillos Overo Negro y Colorado a pradera permitió mejorar significativamente parámetros como peso de canal caliente, área del ojo del lomo, rendimiento centesimal, espesor de grasa dorsal, cobertura de grasa y color muscular, medidos objetiva y subjetivamente; no así el marmoreo. La suplementación con maíz roleado también ayudó a prevenir la presencia de $\mathrm{pH}$ elevado $(>5,8)$ en los novillos; sin embargo, no es posible concluir que esto último se fundamentara en un aumento de las concentraciones de glucógeno muscular registradas en las muestras de tejido muscular post mórtem, ya que éstas no fueron diferentes entre tratamientos. En cuanto a la duración de la suplementación, los resultados no fueron concluyentes y ello probablemente tiene relación con la variabilidad de los factores ambientales no controlables (clima, averías y problemas específicos de cada viaje con animales) cuando se envían animales a matadero. Considerando que la suplementación con maíz roleado tiene un costo relativamente elevado, mientras la industria cárnica chilena no pague un diferencial de calidad en términos de $\mathrm{pH}$ al productor, no será atractivo económicamente usar la suplementación con este propósito.

\section{REFERENCIAS}

Almonacid M. 2003. Estudio de pH y color muscular en cortes comerciales de canales bovinas normales y con la anomalía de "Corte Oscuro". Memoria de título, Escuela de Medicina Veterinaria, Universidad Austral de Chile, Valdivia, Chile.

Amtmann VA, C Gallo, G van Schaik, N Tadich. 2006. Relaciones entre el manejo antemortem, variables sanguíneas indicadoras de estrés y $\mathrm{pH}$ de la canal en novillos. Arch Med Vet 38, 259-264.

AUS - MEAT. 1999. Chiller Assessment Program Standards Manual. AUS Meat Standards, Tingalpa, Australia.

Brown S, E Beavis, P Warriss. 1990. An estimate of the incidence of dark cutting beef in the United Kingdom. Meat Sci 27, 249-258.

Del Campo M, G Brito, JM Soares de Lima, D Vaz Martins, C Sañudo, R San Julián, P Hernández, F Montossi. 2008. Effects of feeding strategies including different proportion of pasture and concentrate, on carcass and meat quality in Uruguayan steers. Meat Sci 80, 753-760.

Ferguson DM, RD Warner. 2008. Have we underestimated the impact of preslaughter stress on meat quality in ruminants? Meat Sci 80, 12-19.

French P, EG Riordan, FJ Monahan, PJ Caffery, MY Mooney, DJ Troy, AP Moloney. 2001. The eating quality of meat of steers fed grass and/or concentrates. Meat Sci 56, 379386.

Gallo C, G Lizondo. 2000. Efectos de diferentes tiempos de ayuno antes del sacrificio sobre el contenido de glucógeno muscular y hepático y el pH final de la canal en novillos. Resumenes del XI Congreso Nacional de Medicina Vete- rinaria, Santiago, Chile.

Gallo C, G Lizondo, T Knowles. 2003. The effects of journey and lairage time on steers transported to slaughter in Chile. Vet $\operatorname{Rec} 152,361-364$.

Gallo C. 2004. Carnes de corte oscuro en bovinos (Dark cutting beef). Revista Americarne para América Latina y El Caribe (español-inglés) 41 (Julio), 10-13.

Gallo C. 2009. Bienestar animal y buenas prácticas de manejo animal relacionadas con la calidad de la carne. En: G Bianchi, Feed O (eds). Introducción a la ciencia de la car$n e .1^{a}$ ed. Editorial Hemisferio Sur, Montevideo, Uruguay, Pp 455-494.

Gardner GE, BL Mclntyre, GD Tudor, DW Pethick. 2001. The impact of nutrition on bovine muscle glycogen metabolism following exercise. Australian J Agric Res 52, 461-470.

Hargreaves A, L Barrales, R Larraín, L Zamorano. 2003. Factores que influyen en el $\mathrm{pH}$ último e incidencia de corte oscuro en canales de bovino. Cienc Inv Agr 3, 155-166.

Hood D, P Tarrant. 1980. The problem of dark cutting in beef. Martinus Nijhoff, The Hague, Netherlands.

Immonen K, M Ruusunen, E Puolanne. 2000ª . Some effects of residual glycogen concentration on the physical and sensory quality of normal pH beef. Meat Sci 55, 33-38.

Immonen K, DM Schaefer, E Puolanne, RG Kauffman, EV Nordheim. $2000^{\mathrm{b}}$. The relative effect of dietary energy density on repleted and resting muscle glycogen concentration. Meat Sci 54, 155-162.

INN, Instituto Nacional de Normalización, Chile. 2002. Canales de bovino. Definiciones y tipificación. Norma Chilena Oficial Nch. 1306, Santiago, Chile.

Johnson ER, Butterfield RM, WJ Priory. 1972. Studies of fat distribution in the bovine carcasses. Australian J Agric Res 23, 381-388.

Knee BW, LJ Cummins, PJ Walker, GA Kearney, RD Warner. 2007. Reducing dark-cutting in pasture fed beef steers by high-energy supplementation. Australian J Exp Agr 47, 1277-1283.

Kover S, H Vos, CA Leede, J Meindertsma. 1984. Meat production from calves depending on genotype and feeding level. Bedrijfsontwkeling 15, 867-869.

Leyva-García IA, F Figueroa-Saavedra, E Sánchez-López, C Pérez-Linares, A Barreras-Serrano. 2012. Impacto económico de la presencia de carne DFD en una planta de sacrificio Tipo Inspección Federal (TIF). Arch Med Vet 44, 39-42.

McVeigh JM, PV Tarrant. 1982. Glycogen content and repletion rates in beef muscle, effects of feeding and fasting. J Nutr 112, 1306-1314.

Mencarini I. 2002. Efectos de dos densidades de carga y dos tiempos de transporte sobre el contenido de glucógeno hepático y muscular, pH y color de la carne. Memoria de título. Escuela de Medicina Veterinaria, Universidad Austral de Chile, Valdivia, Chile.

MINAGRI, Ministerio de Agricultura, Chile. 2009. Reglamento sobre estructura y funcionamiento de mataderos, establecimientos frigoríficos, cámaras frigoríficas y plantas de desposte y fija equipamiento mínimo de tales establecimientos. Decreto No 94 del Ministerio de Agricultura de Chile, Santiago, Chile.

Page JK, DM Wolf, TR Schwortzer. 2001. A survey of beef muscle color and pH. J Anim Sci 79, 678-687.

Pulido R, R Muñoz, P Lemarie, F Wittwer, P Orellana, G Wag- 
horn. 2009. Impact of increasing grain feeding frequency on production of dairy cows grazing pasture. Livest Sci 125, 109-114.

Robelin J, R Daenicke. 1980. Variation of the requirements for cattle growth with liveweight, liveweight, gain, breed and sex. Ann Zootech 29 HS, 15-30.

SAS. SAS User's Guide: Statistics (version 8.1 ed.). 2005. SAS Inst. Inc. Cary NC, USA.
Tarrant PV, J Sherington. 1980. An investigation of ultimate $\mathrm{pH}$ in the muscles of commercial beef carcasses. Meat Sci 4, 287-297.

Vidal R, C Ferrando, A Köpfer, C Almuna. 2009. Pérdidas económicas ocasionadas por corte oscuro en ganado bovino. Resúmenes de la XXXIV Reunión Anual de la Sociedad Chilena de Producción Animal, Pucón, Chile, Pp 242243. 
